\title{
Microbiological Profile of Gastro-Enteritis from Nanded District, Maharashtra State
}

\author{
Dr. Rajhans Vaibhav Vitthalrao ${ }^{1}$, Dr. Raut Sharmila Sanjay ${ }^{2}$, \\ Dr. More Sanjaykumar Rajaram ${ }^{3}$, Dr. Rathod Vimal Shriram ${ }^{4}$, \\ Dr. Gujar Vivek Madhukar ${ }^{5}$, Dr. Kale Chandrahas Damodar ${ }^{6}$, Dr. Kasturi ${ }^{6}$ \\ ${ }^{1}$ Assistant Professor, Microbiology, G.M.C. Aurangabad. \\ ${ }^{2}$ Professor and Head, Microbiology, I.G.G.M.C. Nagpur. \\ ${ }^{3}$ Professor and Head, Microbiology, S.R.T.R.G.M.C. Ambajogai. \\ ${ }^{4}$ Associate Professor, Microbiology, DR.S.C.G.M.C. Nanded. \\ ${ }^{5}$ Associate Professor, Microbiology, Shri V.N.G.M.C. Yavatmal. \\ ${ }^{6}$ Resident Doctors, Microbiology, DR.S.C.G.M.C. Nanded.
}

\section{Introduction}

Microbes are the most significant life forms sharing this planet with humans because of their pervasive presence. ${ }^{1}$ It is estimated that microbes contain $50 \%$ of biological carbon and $90 \%$ of the biological nitrogen on Earth. In terms of sheer number and mass, microbes greatly exceed every other group of organisms on this planet. ${ }^{2}$ There are thousands of different types of microbes that live on and around us, and hundreds that can cause serious human diseases. ${ }^{3}$

Human beings, like other animals, harbor a wide array of microorganisms both on and in their bodies. ${ }^{4}$ It has been estimated that humans have approximately $10^{13}$ cells in their body and about $10^{14}$ bacteria are associated with them. ${ }^{5}$

By the end of $19^{\text {th }}$ century, Koch, among many other scientists had shown that microorganisms were able to cause infectious diseases. ${ }^{6}$ Disease results when the interaction between microbe and human host results in a pathological process. This process is mediated by microbial factors or by the hosts immune response to the presence of the organism ${ }^{5}$ The process of microbial invasion of the body is called "infection". ${ }^{7}$ There are factors relating to the host and environment which are equally important to determine whether or not disease will occur in the exposed host. ${ }^{8}$

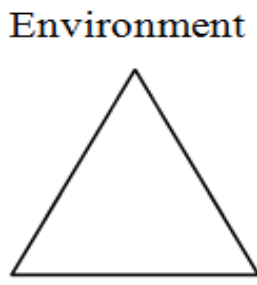

Agent Host

Fig: Epidemiological triad ${ }^{8}$

Micro-organisms can grow on and within other organisms and this microbial colonization sometimes can lead to diseases, disability and even death. Hence the control or destruction of micro-organisms residing within the body of humans is of great importance. ${ }^{2}$ Among such infections, one of the important is Infectious diarrhea which is the second most common cause of death, second only to cardio vascular diseases, worldwide and the leading cause of death in early childhood. ${ }^{9}$ Hence gastroenteritis is an important issue in public health, especially in developing countries. It causes about $11 \%$ of child deaths worldwide, diarrhoea being responsible for 0.7 million deaths in children under 5 years age in $2011^{10}$.

Intestinal parasitic infections have always been an important public health problem in the tropics, particularly in developing countries like India, where the humid climate, the insanitary environment, and poor socioeconomic conditions contribute to the problem. ${ }^{11}$

During the year 2011, about 10.6 million cases of acute diarrhoeal diseases with 1293 deaths were reported in India and they account for about $8 \%$ of deaths in under 5 years age group. ${ }^{12}$

According to a report by National Institute of Cholera and Enteric Disease, Kolkata, (India), crude death rate due to diarrhoea in rural India is 9.3 per 1,000 population and the diarrhoeal deaths account for $22 \%$ of total rural deaths among 0 to 6 years age group children. ${ }^{13}$ In India, one third of the total pediatric admissions 
in hospitals are due to diarrheal diseases and $17 \%$ of all deaths in indoor pediatric patients are diarrhea related. Bacterial causes of childhood diarrhea always exceed the parasitic and fungal pathogens. ${ }^{14}$

Diarrhea is usually defined as 3 or more, loose stools per day, 2 loose stools with abdominal symptoms, or more than 250 grams of stool per day for 7 days. In the U.S., acute gastroenteritis is second only to viral respiratory disease as a cause of acute illness, prompting an estimated 73 million physician consultations each year. ${ }^{9}$

The aetiological spectrum of enteric pathogens causing diarrhoea includes bacteria, parasites, fungi and viruses. ${ }^{15}$ Some of the important bacterial gastroenteric infections include cholera, dysentery, salmonellosis etc. By definition, cholera is endemic when the causative organisms reside in the local environment and the occurrence of the disease in humans is not dependent on the importation of cholera from outside. The mechanism by which cholera becomes endemic depends on the environmental reservoir of cholera. Major outbreaks of cholera usually result from an interplay of factors, such as favorable climate conditions and poor sanitation. ${ }^{16}$ Shigellosis is endemic in India. The isolation rate of Shigella varies from 2-6\% in different studies across India. ${ }^{17} \mathrm{E}$. coli, although a member of normal gastrointestinal microbial flora; is known to cause many pathogenic gastro intestinal conditions ${ }^{4}$

The aim of this study was to find the most common microbial agents responsible for gastroenteritis from Nanded district.

\section{Materials And Methods}

The study was conducted over a period of one year. All the 141 stool samples received at microbiology lab of Dr. S.C.G.M.C. Nanded, during this period were subjected to routine microbiological procedures like microscopy, culture and antibiotic sensitivity testing according to the standard laboratory guidelines.

In case of parasitic infestation, normal saline and iodine preparation were used to demonstrate ova or cyst, accordingly. In cases of suspected cryptosporidial diarrhea, modified Z-N staining was done. ${ }^{18}$

For fungal infections, after the microscopic examination by wet preparation \& gram staining, Sabouraud's Dextrose Agar (SDA) was used for culture. Germ tube test was carried out to differentiate provisionally between Candida albicans and non-albicans. ${ }^{19}$

For bacterial pathogens, Gram staining was used along with wet preparation for morphology and motility. For culture, blood agar, MacConkey agar, Nutrient agar, Alkaline peptone water (APW), Thiosulphate Citrate Bile Sucrose (TCBS) agar, Wilson and Blair's (WB) agar and Deoxycholate Citrate Agar (DCA), Salmonella-Shigella (SS) agar were used.

Bacterial isolates were confirmed by standard Biochemical test procedures and specific antisera. Antibiotic sensitivity testing was done by Kirby- Bauer disc diffusion method using standard antibiotic discs according to the CLSI guidelines, by using antibiotic discs of following concentrations-

Azithromycin (AZM) $15 \mathrm{mcg} / \mathrm{disc}$, Cefpirome (CPM) $30 \mathrm{mcg} / \mathrm{disc}$, Chloramphenicol (C) 30mcg/disc, Ciprofloxacin (CIP) 30mcg/disc, Tetracycline (TE) $30 \mathrm{mcg} / \mathrm{disc}$, Cefazolin (CZ) $30 \mathrm{mcg} / \mathrm{disc}$, Tobramycin (TOB) $10 \mathrm{mcg} / \mathrm{disc}$, Ceftriaxone (CTR) $30 \mathrm{mcg} / \mathrm{disc}$, Cefuroxime (CXM) $30 \mathrm{mcg} / \mathrm{disc}$, Gentamicin (GEN) 10 mcg/disc, Amoxicilin+Clavulanic acid (AMC) 20/10 mcg/disc.

\section{Results}

Out of 141 samples, 40 samples were received only for microscopy and 101 samples were received for microscopy, culture and sensitivity.

Out of 40 samples for microscopy, 17 showed abnormal finding in the form of parasitic ova/cyst. Rest 23 gave non-significant findings.

Out of 101 samples for culture and sensitivity, pathogenic flora was grown in 38 samples, while normal intestinal flora was grown in 63 samples.

In total 141 samples, 55 [39\%] samples showed abnormal findings and rest were normal. Out of 55 samples, 34 [61.81\%] were male and 21 [38.18\%] were female [Fig. No. 1]. Table No.1 shows respective sex wise distribution in affected individuals.

Table No.1 Showing sex wise distribution in microscopy and culture positive cases

\begin{tabular}{|l|l|l|l|}
\hline & Microscopy & Culture & Total \\
\hline Male & 8 & 26 & $\mathbf{3 4}$ \\
\hline Female & 9 & 12 & $\mathbf{2 1}$ \\
\hline Total & $\mathbf{1 7}$ & $\mathbf{3 8}$ & $\mathbf{5 5}$ \\
\hline
\end{tabular}

Fig. No.1 Showing sex wise distribution of stool samples which showed significant findings in microscopy or culture 


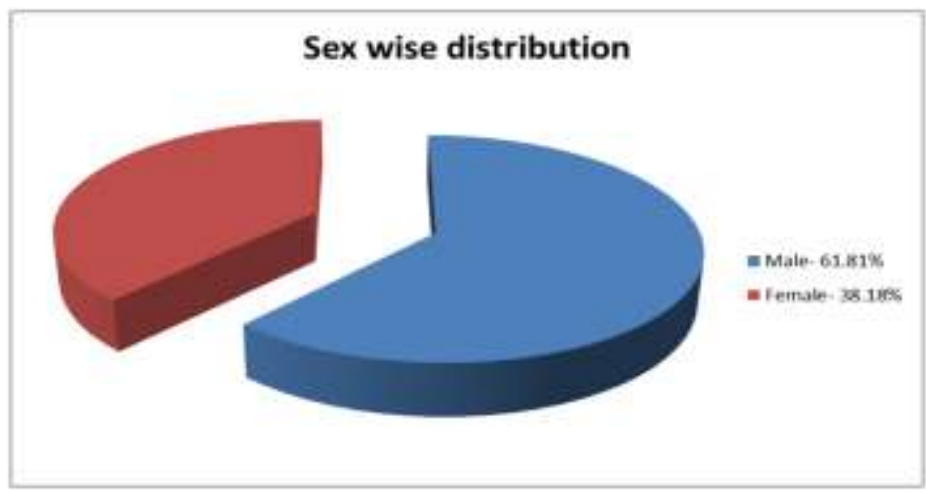

Out of 38 bacterial isolates, Shigella spp. was most common i.e. in 12 [31.57\%] samples. Vibrio cholerae and E.coli were isolated in 9 [23.68\%] samples each. Staphylococcus aureus was isolated from 3 [7.89\%] samples, Citrobactor spp.from 2 [5.26\%] samples; and Salmonella paratyphi A, Klebsiella pneumonie, Pseudomonas aeruginosa, Coagulase Negative Staphylococcus (CoNS) were isolated from 1 [2.63\%] stool sample each. Table No.2 shows the distribution of bacterial isolates.

Table No.2 Showing distribution of bacterial isolates

\begin{tabular}{|l|l|l|}
\hline Bacteria isolated & No of samples & Percentage \\
\hline Shigella spp. & 12 & 31.57 \\
\hline Vibrio cholerae Eltor Ogawa & 9 & 23.68 \\
\hline E.Coli & 9 & 23.68 \\
\hline Staphylococcus aureus & 3 & 7.89 \\
\hline Citrobactor spp. & 2 & 5.26 \\
\hline Salmonella paratyphi A & 1 & 2.63 \\
\hline Klebsiella pneumonie & 1 & 2.63 \\
\hline Pseudomonas aeruginosa & 1 & 2.63 \\
\hline CoNS & 1 & 2.63 \\
\hline Total & $\mathbf{3 8}$ & $\mathbf{1 0 0}$ \\
\hline
\end{tabular}

Among parasites and fungi, oocyst of Cryptosporidium parvum was observed in maximum i.e. 8 [47.05\%] samples. Fertilised egg of Ascaris lumbricoides [Round worm], egg of Ancylostoma duodenale [Hook worm] and larva of Strongyloides sterocoralis were isolated from 1 [5.88\%] stool sample each.

On the other hand, 6 [35.29\%] samples showed presence of budding yeast cells. After culture on SDA, 2 of those 6 gave positive Germ Tube Test [GTT], rest 4 being negative for GTT. Table No.3 shows the parasitic and fungal findings [Fig. No. 2].

Table No.3 Showing distribution of parasites, their ova/cyst and fungi by microscopy

\begin{tabular}{|l|l|l|}
\hline Parasite Observed & No of samples & Percentage \\
\hline Oocyst of Cryptosporidium parvum & 8 & 47.05 \\
\hline Budding yeast cells & 6 & 35.29 \\
\hline Fertilised egg of Ascaris & 1 & 5.88 \\
\hline Egg of Ancylostma & 1 & 5.88 \\
\hline Larva of Strongyloides & 1 & 5.88 \\
\hline Total & $\mathbf{1 7}$ & $\mathbf{1 0 0}$ \\
\hline
\end{tabular}

Fig. No.2 Showing distribution of parasites, their ova/cyst and fungi by microscopy

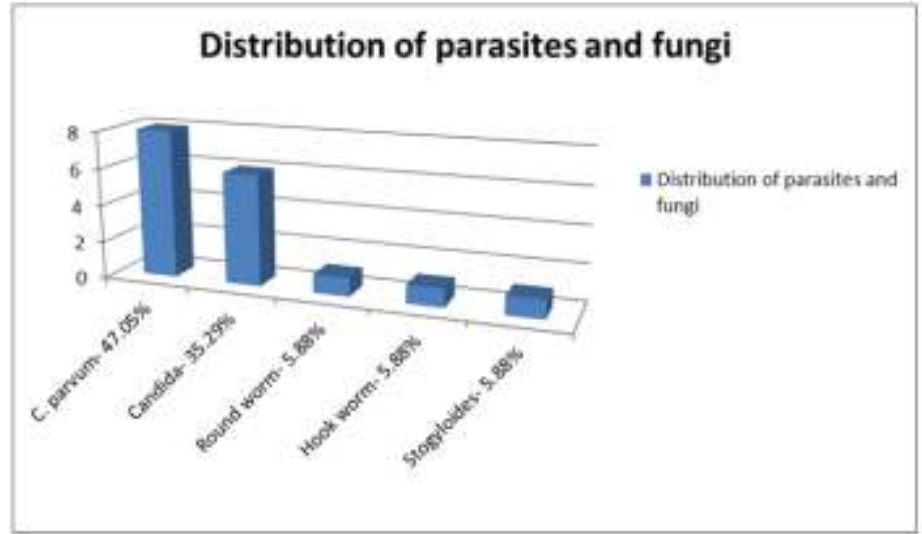

Antibiotic susceptibility testing

Table No. 4: Antibiotic susceptibility pattern of gram negative bacilli 
Microbiological Profile Of Gastro-Enteritis From Nanded District, Maharashtra State

\begin{tabular}{|l|l|l|l|l|l|l|l|l|l|}
\hline \multirow{2}{*}{ Type of Bacteria } & \multicolumn{7}{|c|}{ Antibiotic sensitivity (In Percentage \% ) } \\
\cline { 2 - 12 } & C & CIP & TE & TOB & GEN & CPM & CZ & AMC & CXM \\
\hline Shigella spp $(\mathbf{n = 1 2})$ & 75 & 91.6 & 0 & 100 & 75 & 100 & 50 & 75 & 50 \\
\hline Vibrio cholerae $(\mathbf{n}=\mathbf{9})$ & 100 & 100 & 100 & 66.6 & 33.3 & 88.8 & 55.5 & 100 & 44.4 \\
\hline E.coli $(\mathbf{n}=\mathbf{9})$ & 88.8 & 77.7 & 100 & 100 & 100 & 0 & 33.3 & 33.3 & 22 \\
\hline Citrobactor spp $(\mathbf{n}=\mathbf{2})$ & 100 & 100 & 50 & 100 & 100 & 0 & 50 & 100 & 50 \\
\hline Salmonella paratyphi $\boldsymbol{A}(\mathbf{n}=\mathbf{1})$ & 100 & 100 & 100 & 0 & 100 & 0 & 100 & 0 & 100 \\
\hline Klebsiella pnemoniea $(\mathbf{n}=\mathbf{1})$ & 100 & 0 & 100 & 0 & 100 & 100 & 100 & 0 & 0 \\
\hline
\end{tabular}

Among Shigella, most of the isolates were uniformly sensitive to Cefpirome (100\%) and then to Chloramphenicol (75\%). All the isolates were uniformly resistant to Tetracycline $(0 \%)$

Among Vibrio cholerae, all isolates were uniformly sensitive to Chloramphenicol (100\%) and Tetracycline (100\%) Almost all strains were resistant to Cefuroxime (11\%)

Among E.coli isolates, all were uniformly sensitive to Tobramycine $(100 \%)$ and almost all were resistant to Cefuroxime (22\%)

Salmonella paratyphi A was sensitive to Ceftriaxone, Cephazolin, Gentamicine, Tetracycline, Chloramphenicol, Ciprofloxacin and resistant to Tobramycine and Amoxicilin+Clavulanic acid.

\section{Discussion}

Although an important health issue affecting all the age groups, very few studies have been done comprising all the pathogens causing gastro enteritis like bacteria, fungi and parasites.

Fricker J studied frequently identified pathogens in children with acute diarrhea in developing countries during the Year 1990 and $2000^{20}$ According to this study, Shigella was observed to be isolated in $5-15 \%$ of cases, Vibrio cholerae $\mathrm{O} 1$ in 5-10\% and Salmonella in 1-5\% of cases. Total isolation rate of E.coli was around $10-25 \%$ and Cryptosporidium parvum was found in 5-15\% of cases.

In comparison to this study, Shigella and Vibrio were isolated more frequently than E.coli, in our study. This higher isolation rate might be contributed to the sudden outbreaks, which were encountered during our study period. Isolation of Salmonella was marginally lower in our study.

VyasKumar Rathaur et al in their study mention Salmonella isolation rate as $13.6 \%$ in 280 samples. $^{21}$ This was also higher as compared to our study.

In a 15 years study done at MGIMS, Wardha, India, average isolation rate of Vibrio cholera was observed to be $5.14 \%$, range of isolation rate per year being $1.2 \%$ to $10.53 \%{ }^{22}$. The isolation rate observed in our study is higher which might be due to the outbreaks, same as in case of Shigella; almost all cases being reported from same locality, same time.

In a bacteriological analysis of acute diarrhea cases at a surveillance site and in different diarrhea outbreak areas of Orissa from May to October 2005, Out of 265 culture-positive samples, Vibrio cholerae was isolated in 56 samples $(20.8 \%)^{23}$ which is comparable to our study.

Ravinder Kaur et al ${ }^{11}$ observed similar isolations to our study, i.e. Cryptosporidium spp in 24 [40.7\%] samples and Ascaris lumbricoides in 1 [1.7\%] samples. These findings are comparable to our study.

Although antibiotics play a little role in gastro-intestinal infections, there importance can never be denied. Prescription of empirical antibiotic treatment requires a balance between the detriments associated with the use of broad-spectrum antibiotic drugs (cost, side-effects and impact on future resistance) and the increased chance of matching the in vitro susceptibility of the pathogen.

Antibiotic drugs account for $20-50 \%$ of a hospital's pharmaceutical expenditure. Resistance to antibiotics is a major problem, and strains of bacteria resistant to almost all drugs are emerging. One must therefore ask whether, to balance this, there is a clear benefit to the use of an appropriate antibiotic drug during the 24-48 hours before the results of cultures are available and how significant is this benefit. ${ }^{24}$

There is a need to integrate medicine and innovative technology in our public health system to provide rapid, efficient, accurate, and cost-effective results for identification and antimicrobial susceptibility testing (AST) of pathogens. Automated identification/AST systems can aid in rapid diagnosis of bacterial pathogens. ${ }^{25}$

\section{Conclusion}

In this study, males $(61.8 \%)$ were affected more than females $(38.1 \%)$. Most common parasitic infestation was that of Cryptosporidium parvum (47\%) which was followed by round worm (5.8\%) and hook worm (5.8\%) each.

Bacteriological study revealed the most common cause of gastro enteritis was due to Shigella species (31.5\%) among bacteria. The next being Vibrio cholerae (23.6\%) sharing its place with E.coli (23.6\%)

The case history shows that, majority of these cases had presented from close locality or same family and within a short time period, highlighting the mode of transmission i.e. feco-oral route and through 
contaminated water and food. Hence through this study, the need of some simply basic but practically effective measures is underlined-

\section{$\checkmark$ Proper disposal of sewage \\ $\checkmark$ Regular and proper hand washing; at least before eating and after toilet \\ $\checkmark$ Eating healthy, clean food \\ $\checkmark$ Provision of safe drinking water.}

[1]. Parija SC. Textbook of Microbiology. 2nd ed. $619 \mathrm{p}$.

[2]. Willey, Sherwood, Woolverton. Microbiology. 7th ed. McGraw-Hill; 1 p.

[3]. Murray, Rosenthal, Pfaller. Medical Microbiology. 1st ed. Elsevier Mosby; 1 p.

[4]. R Ananthanarayan Paniker CKJ. Textbook of Microbiology. 9th Editio. Arti Kapil, editor. Chennai: Universities Press; 2013.

[5]. Parija SC. Textbook of Microbiology \& Immunology. 2nd ed. Elsevier; 3 p.

[6]. Koneman E. Colour atlas \& Textbook of diagnostic Microbiology. 5th ed. Kosby; 2 p.

[7]. Greenwood D. Midical Microbiology. 16th ed. Churchil LivingstonePublications; 3 p.

[8]. Park K. Park's Textbook of Preventive \& Social Medicine. 22nd ed. Bhanot Publications; $31 \mathrm{p}$.

[9]. Dr Charles Bryan. Gastrointestinal and intra-abdominal infections. In: University of South Carolina School of Medicine, editor. Infectious disease.

[10]. Report P. Committing to Child Survival : A Promise Renewed. 2012.

[11]. Kaur R, Rawat D, Kakkar M, Uppal B SV. Intestinal parasites in children with diarrhea in Delhi, India. Southeast asian j trop med public Heal. 33(4):725-7.

[12]. DGHS, Central bureau of Health Intelligence, Minisry of Health and Family Welfare ND. National Health profile 2011 (Jan-Dec). New Delhi; 2011.

[13]. Estimation of the Burden of Diarrhoeal diseases in India, NICED Kolkata available from http://www.whoindia.org/linkfiles/commision on macroeconomic and health Bg P2 Estimation of the burden of diarrhoeal diseases in India.Pdf.

[14]. Das S, Saha R SS. Enteric pathogens in north Indian patients with diarrhoea. Indian J Community Med. 2007;32:27-31.

[15]. Namita A. Raytekar, Santosh Saini SD. Intestinal parasitic prevalence in human immuno-deficient virus (HIV) infected patients with and without diarrhoea and its Association with CD4 T cells counts. Int J Biomed Adv Res. 2012;3(11):853-7.

[16]. Kanungo S, Sah BK, Lopez AL, Sung JS, Paisley AM, Sur D, Clemens JD NG. Cholera in India: an analysis of reports, 1997-2006. Bull World Health Organ. 2010;88:185-91.

[17]. Nath R, Saikia L, Choudhury G, Sharma D. Drug resistant Shigella flexneri in \& around Dibrugarh, north-east India. Indian J Med Res. 2013;137(1):183-6.

[18]. Chatterjee KD. Parasitology (Protozoology and Helminthology). 13 Ed. Kolkata: CBS publishers and distributers pvt. ltd.;

[19]. Chander J. Textbook of Medical Mycology. 3rd Ed. New Delhi: Mehta Publishers;

[20]. Fricker J. Children in tropics. 1993.

[21]. VyasKumar Rathaur, Monika Pathania, Aparna Jayara, NeerajYadav, Clinical Study of Acute Childhood Diarrhoea Caused by Bacterial Enteropathogens, Journal of Clinical and Diagnostic Research. 2014 May, Vol-8(5): PC01-PC05.

[22]. Narang P, Mendiratta D, Deotale V, Narang R. Changing patterns of vibrio cholerae in Sewagram between 1990 and 2005. Indian J Med Microbiol. 2008;26(1):40-4.

[23]. Pal B, Khuntia H, Samal S, Das S, Chhotray G. Emergence of Vibrio cholerae O1 biotype El Tor serotype Inaba causing outbreaks of cholera in Orissa, India. India Jpn J Infect Dis. 2006;59:266-9.

[24]. Leibovici, Shraga, Drucker, Konigsberger, Samra, Pitlik. The benefit of appropriate empirical antibiotic treatment in patients with bloodstream infection. J Intern Med. 1998;244(5):379-86.

[25]. Duggal S, Gaind R, Tandon N, Deb M, Chugh TD. Comparison of an Automated System with Conventional Identification and Antimicrobial Susceptibility Testing, ISRN Microbiology, Volume 2012 (2012), Article ID 107203, 4 pages, http://dx.doi.org/10.5402/2012/10.

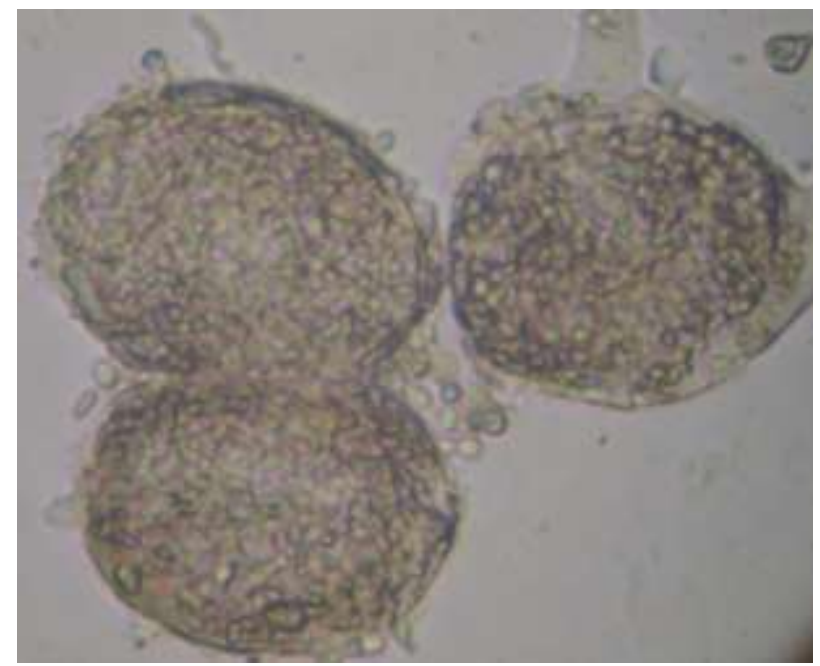

Photo No. 1 Unfertilized eggs of Ascaris lumbricoides along with budding yeast cells [40x] 


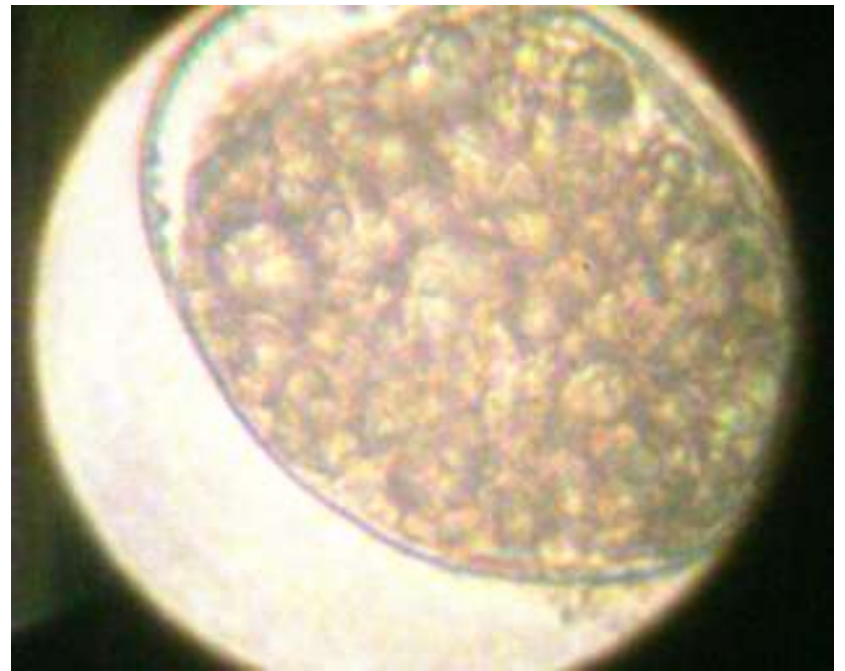

Photo No. 2- Unfertilized egg of Ascaris lumbricoides [40x] 